\title{
Effects Oof Illness Perceptions On Health-Related Quality of Life in Patients With Rheumatoid Arthritis in China
}

\section{Zhaohui Zheng ( $\nabla$ zhengzhimmu@163.com )}

Fourth Military Medical University https://orcid.org/0000-0001-7804-4474

Juan Wang

Fourth Military Medical University

\section{Zhe Yang}

Fourth Military Medical University

\section{Yan Zheng}

Fourth Military Medical University

Yaling Peng

Fourth Military Medical University

\section{Qing Wang}

Fourth Military Medical University

\section{Hongli Xia}

Fourth Military Medical University

\section{Yan Wang}

Fourth Military Medical University

Jin Ding

Fourth Military Medical University

\section{Ping Zhu}

Fourth Military Medical University

Lei Shang

Fourth Military Medical University

\section{Research}

Keywords: rheumatoid arthritis, illness perceptions, health-related quality of life (HRQoL), SF-36, correlation analysis,

Posted Date: September 30th, 2020

DOI: https://doi.org/10.21203/rs.3.rs-71405/v1

License: (c) (i) This work is licensed under a Creative Commons Attribution 4.0 International License. Read Full License

Version of Record: A version of this preprint was published at Health and Quality of Life Outcomes on April 20th, 2021. See the published version at https://doi.org/10.1186/s12955-021-01770-4. 


\section{Abstract}

Objectives: For patients with rheumatoid arthritis (RA) in China, little is known of how their illness perceptions affect their health-related quality of life (HRQoL). The present study detailed associations between characteristics of illness perception and features of HRQoL.

Methods: For 191 patients with RA, illness perceptions were measured using the Brief Illness Perceptions Questionnaire (BIPQ) with 8 domains. HRQoL was determined with the Medical Outcomes Study 36-Item Short-Form Health Survey (SF-36). The independent samples $t$-test, one-way analysis of variance, linear regression analyses, and multivariate stepwise regression was used to analyze these data.

Results: The overall BIPQ of patients with RA was $49.09 \pm 11.06$. The highest and lowest scores were for concern $(9.15 \pm 1.81)$ and personal control (4.30 \pm 2.52$)$, respectively. Multivariate stepwise regression analyses showed that domains of BIPQ including consequences, personal control, treatment control, identity, coherence, emotional response, and the overall BIPQ score were associated with HRQoL, identity and emotional response were negatively associated with HRQoL but personal control, treatment control positively $(P<0.05)$. Meanwhile, the overall BIPQ had a significantly negative association with each component and the summary scores of HRQoL.

Conclusions: Illness perceptions were associated with HRQoL of patients with RA in China. Illness perceptions were identified as likely targets for strategies to improve quality of life in patients with RA in China. This study provides evidence that can help improve HRQoL in patients with RA.

\section{Introduction}

Rheumatoid arthritis (RA) is a chronic disease characterized by symmetric polyarticular arthritis. It is the most common autoimmune disease. Clinical manifestations of RA include joint swelling, pain, and limited function [1,2]. Progression of RA may eventually lead to joint deformities which seriously affect patients' quality of life[3, 4]. In RA, each patient's presentation and course of disease is individual. In recent years, there has been growing interest in a common sense model and its application in research on chronic diseases. With a common sense model, illness perception is the key construct that suggests that people build their own cognitive and emotional representations of illness, which are the basis for how they respond to their illness[5-9]. Individuals actively try to make sense of symptoms, forming personal beliefs about their illness, which, in turn, determine their subsequent coping behavior and quality of life[10].

Health-related quality of life (HRQoL) may be measured to reveal the physiological, psychological, and social functions of patients with RA[3, 11 , 12]. Studies have shown that illness perception is associated with quality of life, social function, and disease prognosis[13-18]. Such knowledge is applied to guide clinicians in the management of RA, to improve the HRQoL and the prognosis of their patients. In rheumatology, the effect of illness perception has been studied extensively in western populations, specifically for RA[19, 20], systemic sclerosis [21], lupus nephritis[22, 23], psoriatic arthritis[16], multiple sclerosis[24], and systemic lupus erythematosus[25, 26]. Research has highlighted the importance of the beliefs of patients with RA about their illness and symptoms as they affect their HRQoL. The identification of these patients' perceptions could positively influence quality of life, as illness perception is amenable to intervention[27].

However, to our knowledge, there have been no studies on illness perception and its association with HRQoL for patients with RA in China. To provide a reference for improving the HRQoL of patients with RA, the present study investigated the association between illness perceptions and HRQoL of patients with RA in China. The results of our study will provide foundational evidence to improve the identification of patients' perceptions of illness and quality of life, better guide clinicians in management of RA, and improve the ability of patients with RA to self-manage and improve their HRQoL.

\section{Methods}

\subsection{Study design and data collection}

This cross-sectional study was conducted in an outpatient clinic at Xijing Hospital, Xi'an, Shaanxi, China in the year 2017. The Ethics Committee of Xijing Hospital approved the study (KY20140902-5), and all subjects provided written informed consent prior to participate. The study included patients with RA diagnosed according to the ACR (American College of Rheumatology)/EULAR (European League against Rheumatism) 2009 classification criteria; $\geq 18$-years-old; able to understand and communicate in Chinese; and willing to participate. Patients with any of the following were excluded: suffering from other chronic diseases; recent major surgery; unstable condition; or intellectual or cognitive impairments.

Face-to-face interviews with the patients were conducted. Sociodemographic and clinical data were collected. Disease activity estimates were based on the Disease Activity Score 28 (DAS28). The DAS28 is an index that comprises an inflammatory biomarker (erythrocyte sedimentation rate or C-reactive protein [CRP]), and physician-rated tenderness and swelling score of 28 joints. The DAS28 specifically is based on the number 
of tender and swollen joints, CRP, and patient global assessment[28]. Illness perceptions and HRQoL (see below) were assessed via patientreported outcome measures.

\subsection{Measurements of illness perceptions}

The Brief IIIness Perceptions Questionnaire (BIPQ) assesses an individual's perceptions and cognitions regarding their disease [7, 29]. The Chinese BIPQ has been tested and validated previously[30, 31]. The questionnaire measures the following 8 domains of illness perception: consequences; timeline; personal control; treatment control; identity; concern; coherence; and emotional response. The overall BIPQ score ranges from 0 to 80; a higher score reflects a more negative view of the illness.

\subsection{Measurements of HRQoL}

HRQoL was measured with the Chinese version of the Medical Outcomes Study 36-item Short-Form Health Survey (SF-36). The SF-36 consists of 36 items that measured the following 8 dimensions: physical function (PF); role limitations related to physical problems (RP); bodily pain (BP); general health perception (GH); vitality (VT); social functioning (SF); role limitations due to emotional problems (RE); and mental health $(\mathrm{MH})$. The score of each dimension is converted to a standard score ranging from 0 to 100, with a higher score indicating better HRQoL [32]. The SF-36 has showed good reliability and validity among various Chinese patient populations[33, 34].

\subsection{Statistical analysis}

Statistical analyses were performed using Statistical Package for Social Science 18.0 (SPSS, Chicago, IL) software. The descriptive statistics are presented as the mean and standard deviation for quantitative data, and percentage for count data. The independent samples $t$-test and one-way analysis of variance were used to analyze inter-group differences with normal distribution.

Linear regression analyses were used to test the univariate correlations between the domains of illness perception and HRQoL, and to screen the significant independent variables $(P<0.1)$ for subsequent multivariate regression analyses. Multivariate stepwise regression analysis was used to explore the effect of illness perceptions on HRQoL. After controlling for demographics and disease characteristics, independent variables were entered into the model stepwise. $P$ values $<0.1$ were added into the regression, and only $P$ values $<0.05$ were considered statistically significant.

\section{Results}

\subsection{Patient characteristics}

The questionnaires were distributed to 200 eligible patients, and 9 with missing data were excluded from the final analysis; thus 200 sets of surveys were distributed and 191 were completed (response rate 95.5\%). Of the 191 study participants, the average age of the patients was $45.06 \pm 13.32$ years, and 140 (73.30\%) were women (Table 1). Of the 191 participants, $37.7 \%$ had a disease duration of more than 5 years, $26.18 \%$ were in remission, and $16.75 \%$ had severe disease. 
Table 1

Baseline characteristics of the patients $(n=191)^{a}$

\begin{tabular}{|c|c|c|}
\hline \multirow[t]{2}{*}{ Gender } & Male & $51(26.70)$ \\
\hline & Female & $140(73.30)$ \\
\hline \multicolumn{2}{|l|}{ Age, y } & $45.06 \pm 13.32$ \\
\hline \multirow[t]{3}{*}{ Ethnic group } & Han & $182(95.29)$ \\
\hline & Hui & $8(4.19)$ \\
\hline & Other & $1(0.52)$ \\
\hline \multirow[t]{2}{*}{ Marital status } & Married & $168(87.96)$ \\
\hline & Other & $23(12.04)$ \\
\hline \multirow[t]{3}{*}{ Education } & $\leq$ Junior middle school (9 y) & $82(42.93)$ \\
\hline & Senior middle school (12 y) & $41(21.47)$ \\
\hline & $\geq$ College $(15 y)$ & $68(35.60)$ \\
\hline \multirow[t]{2}{*}{ Residence } & Rural & $95(49.74)$ \\
\hline & Urban & $96(50.26)$ \\
\hline \multirow[t]{3}{*}{ Income/month, yuan b } & under 1000yuan & $45(23.56)$ \\
\hline & $1000-3000$ yuan & $94(49.21)$ \\
\hline & above 3000 yuan & $52(27.23)$ \\
\hline \multirow[t]{3}{*}{ Occupation } & Unemployed & 76 (39.79) \\
\hline & Blue collar & $39(20.42)$ \\
\hline & White collar & $76(39.79)$ \\
\hline \multicolumn{2}{|l|}{ Disease duration, y } & $5.73 \pm 7.33$ \\
\hline \multicolumn{2}{|l|}{ DAS28 } & $3.58 \pm 1.32$ \\
\hline \multicolumn{3}{|c|}{ a Reported as $n(\%)$ unless indicated otherwise. } \\
\hline \multicolumn{3}{|c|}{ b Family income /monthly. } \\
\hline
\end{tabular}

\subsection{Illness perceptions}

The BIPQ comprises 8 domains that together reflect an individual's perception of their disease. The score of each domain may range from 0 to 10 for a total maximum of 80 , with the highest score registering the greatest aggravation.

The overall BIPQ score of the participants was $49.09 \pm 11.06$. The scores of each dimension of the BIPQ were as follows: concern (9.15 \pm 1.81$)$; timeline (7.68 \pm 2.78$)$; treatment control (7.83 \pm 2.3$)$; emotions (7.15 \pm 2.9$)$; consequences (6.72 \pm 3.02$)$; identity (6.61 \pm 2.74$)$; coherence (6.08 \pm $2.60)$; and personal control $(4.30 \pm 2.52)$. Concern and timeline received the highest, while personal control had the lowest.

\subsection{HRQoL}

HRQoL was measured with the SF-36. The scores of each of the 8 dimensions tested (i.e., physical function, role limitations related to physical problems, bodily pain, general health perception, vitality, social functioning, role limitations due to emotional problems, and mental health) could range from 0 to 100, with a highest score indicating best HRQoL (Table 2). 
Table 2

SF-36 scores for 8 dimensions reflecting HRQoL ${ }^{a}$ by various population stratifications $(n=191)$

\begin{tabular}{|c|c|c|c|c|c|c|c|c|c|}
\hline & & PF & RP & BP & GH & VT & SF & RE & $\mathrm{MH}$ \\
\hline \multirow[t]{2}{*}{ Gender } & Male & $\begin{array}{l}54.22 \\
\pm \\
33.46\end{array}$ & $\begin{array}{l}19.61 \pm \\
35.47\end{array}$ & $\begin{array}{l}41.96 \pm \\
26.79\end{array}$ & $\begin{array}{l}34.08 \pm \\
20.72\end{array}$ & $\begin{array}{l}45.88 \pm \\
24.08\end{array}$ & $\begin{array}{l}70.34 \pm \\
32.30\end{array}$ & $\begin{array}{l}25.49 \pm \\
40.87\end{array}$ & $\begin{array}{l}62.12 \pm \\
21.25\end{array}$ \\
\hline & Female & $\begin{array}{l}60.11 \\
\pm \\
27.80\end{array}$ & $\begin{array}{l}23.21 \pm \\
37.17\end{array}$ & $\begin{array}{l}47.39 \pm \\
26.16\end{array}$ & $\begin{array}{l}37.67 \pm \\
20.32\end{array}$ & $\begin{array}{l}52.75 \pm \\
22.71\end{array}$ & $\begin{array}{l}76.61 \pm \\
31.91\end{array}$ & $\begin{array}{l}33.10 \pm \\
40.87\end{array}$ & $\begin{array}{l}60.40 \pm \\
23.41\end{array}$ \\
\hline \multirow[t]{2}{*}{ Age, y } & $<45$ & $\begin{array}{l}69.04 \\
\pm \\
24.53 \\
b\end{array}$ & $\begin{array}{l}25.28 \pm \\
36.05\end{array}$ & $\begin{array}{l}49.29 \pm \\
23.14\end{array}$ & $\begin{array}{l}39.47 \pm \\
18.64\end{array}$ & $\begin{array}{l}55.17 \pm \\
21.25^{b}\end{array}$ & $\begin{array}{l}80.06 \pm \\
28.75^{b}\end{array}$ & $\begin{array}{l}34.08 \pm \\
40.19\end{array}$ & $\begin{array}{l}61.84 \pm \\
21.86\end{array}$ \\
\hline & $\geq 45$ & $\begin{array}{l}49.36 \\
\pm \\
30.34\end{array}$ & $\begin{array}{l}19.61 \pm \\
37.17\end{array}$ & $\begin{array}{l}43.01 \pm \\
28.69\end{array}$ & $\begin{array}{l}34.30 \pm \\
21.68\end{array}$ & $\begin{array}{l}47.21 \pm \\
24.32\end{array}$ & $\begin{array}{l}70.47 \pm \\
34.19\end{array}$ & $\begin{array}{l}28.43 \pm \\
41.53\end{array}$ & $\begin{array}{l}60.00 \pm \\
23.69\end{array}$ \\
\hline \multirow[t]{3}{*}{ Education } & $\begin{array}{l}\leq \text { Junior } \\
\text { middle } \\
\text { school }\end{array}$ & $\begin{array}{l}46.71 \\
\pm \\
29.88 \\
b\end{array}$ & $\begin{array}{l}14.63 \pm \\
33.09^{b}\end{array}$ & $\begin{array}{l}36.32 \pm \\
25.81^{b}\end{array}$ & $\begin{array}{l}31.71 \pm \\
22.34^{b}\end{array}$ & $\begin{array}{l}42.20 \pm \\
25.03^{b}\end{array}$ & $\begin{array}{l}64.33 \pm \\
34.31^{b}\end{array}$ & $\begin{array}{l}23.17 \pm \\
37.29\end{array}$ & $\begin{array}{l}52.59 \pm \\
24.81^{b}\end{array}$ \\
\hline & $\begin{array}{l}\text { Senior } \\
\text { middle } \\
\text { school }\end{array}$ & $\begin{array}{l}62.32 \\
\pm \\
29.63\end{array}$ & $\begin{array}{l}26.22 \pm \\
39.51\end{array}$ & $\begin{array}{l}47.59 \pm \\
26.64\end{array}$ & $\begin{array}{l}40.37 \pm \\
20.89\end{array}$ & $\begin{array}{l}56.95 \pm \\
19.97\end{array}$ & $\begin{array}{l}80.18 \pm \\
29.04\end{array}$ & $\begin{array}{l}36.59 \pm \\
46.43\end{array}$ & $\begin{array}{l}68.10 \pm \\
21.46\end{array}$ \\
\hline & $\geq$ College & $\begin{array}{l}70.51 \\
\pm \\
23.01\end{array}$ & $\begin{array}{l}29.04 \pm \\
37.81\end{array}$ & $\begin{array}{l}56.54 \pm \\
22.67\end{array}$ & $\begin{array}{l}40.54 \pm \\
16.33\end{array}$ & $\begin{array}{l}57.79 \pm \\
19.25\end{array}$ & $\begin{array}{l}84.56 \pm \\
27.16\end{array}$ & $\begin{array}{l}37.25 \pm \\
40.53\end{array}$ & $\begin{array}{l}66.47 \pm \\
17.58\end{array}$ \\
\hline \multirow[t]{2}{*}{ Residence } & Rural & $\begin{array}{l}53.84 \\
\pm \\
30.20\end{array}$ & $\begin{array}{l}20.79 \pm \\
36.04\end{array}$ & $\begin{array}{l}39.97 \pm \\
25.61^{b}\end{array}$ & $\begin{array}{l}33.25 \pm \\
21.54^{b}\end{array}$ & $\begin{array}{l}47.58 \pm \\
25.33\end{array}$ & $\begin{array}{l}71.57 \pm \\
35.84\end{array}$ & $\begin{array}{l}31.23 \pm \\
42.33\end{array}$ & $\begin{array}{l}57.09 \pm \\
25.11^{b}\end{array}$ \\
\hline & Urban & $\begin{array}{l}63.18 \\
\pm \\
28.06\end{array}$ & $\begin{array}{l}23.70 \pm \\
37.41\end{array}$ & $\begin{array}{l}51.84 \pm \\
25.90\end{array}$ & $\begin{array}{l}40.14 \pm \\
18.77\end{array}$ & $\begin{array}{l}54.22 \pm \\
20.54\end{array}$ & $\begin{array}{l}78.26 \pm \\
27.58\end{array}$ & $\begin{array}{l}30.90 \pm \\
39.66\end{array}$ & $\begin{array}{l}64.58 \pm \\
19.72\end{array}$ \\
\hline \multirow[t]{3}{*}{$\begin{array}{l}\text { Income/month, } \\
\text { yuan }^{c}\end{array}$} & $<1000$ & $\begin{array}{l}47.89 \pm \\
32.66^{b}\end{array}$ & $\begin{array}{l}13.33 \pm \\
32.25\end{array}$ & $\begin{array}{l}38.42 \pm \\
26.51^{b}\end{array}$ & $\begin{array}{l}31.13 \pm \\
24.78\end{array}$ & $\begin{array}{l}41.67 \pm \\
25.02^{b}\end{array}$ & $\begin{array}{l}64.72 \pm \\
34.78^{b}\end{array}$ & $\begin{array}{l}23.70 \pm \\
40.59\end{array}$ & $\begin{array}{l}53.96 \\
\pm \\
26.09 \\
b\end{array}$ \\
\hline & $1000-3000$ & $\begin{array}{l}59.57 \\
\pm \\
27.50\end{array}$ & $\begin{array}{l}23.40 \pm \\
37.35\end{array}$ & $\begin{array}{l}44.35 \pm \\
25.27\end{array}$ & $\begin{array}{l}36.99 \pm \\
19.09\end{array}$ & $\begin{array}{l}52.39 \pm \\
22.51\end{array}$ & $\begin{array}{l}76.33 \pm \\
32.56\end{array}$ & $\begin{array}{l}30.85 \pm \\
40.08\end{array}$ & $\begin{array}{l}61.91 \pm \\
21.99\end{array}$ \\
\hline & $>3000$ & $\begin{array}{l}65.87 \\
\pm \\
27.81\end{array}$ & $\begin{array}{l}27.88 \pm \\
38.24\end{array}$ & $\begin{array}{l}55.31 \pm \\
25.99\end{array}$ & $\begin{array}{l}41.04 \pm \\
17.73\end{array}$ & $\begin{array}{l}56.25 \pm \\
20.88\end{array}$ & $\begin{array}{l}81.25 \pm \\
26.72\end{array}$ & $\begin{array}{l}37.82 \pm \\
42.28\end{array}$ & $\begin{array}{l}64.92 \pm \\
20.24\end{array}$ \\
\hline \multirow[t]{3}{*}{ Occupation } & Unemployed & $\begin{array}{l}48.36 \\
\pm \\
28.69 \\
b\end{array}$ & $\begin{array}{l}13.49 \pm \\
32.26^{b}\end{array}$ & $\begin{array}{l}37.93 \pm \\
27.00^{b}\end{array}$ & $\begin{array}{l}32.29 \pm \\
20.26^{b}\end{array}$ & $\begin{array}{l}43.82 \pm \\
24.00^{b}\end{array}$ & $\begin{array}{l}67.11 \pm \\
34.45^{b}\end{array}$ & $\begin{array}{l}23.68 \pm \\
38.80\end{array}$ & $\begin{array}{l}55.21^{ \pm} \\
25.91^{b}\end{array}$ \\
\hline & Blue collar & $\begin{array}{l}59.49 \\
\pm \\
29.49\end{array}$ & $\begin{array}{l}19.87 \pm \\
34.02\end{array}$ & $\begin{array}{l}39.67 \pm \\
24.63\end{array}$ & $\begin{array}{l}35.13 \pm \\
24.12\end{array}$ & $\begin{array}{l}49.10 \pm \\
23.48\end{array}$ & $\begin{array}{l}70.19 \pm \\
32.77\end{array}$ & $\begin{array}{l}32.48 \pm \\
42.22\end{array}$ & $\begin{array}{l}57.54 \pm \\
22.06\end{array}$ \\
\hline & White collar & $\begin{array}{l}68.22 \\
\pm \\
27.05\end{array}$ & $\begin{array}{l}32.24 \pm \\
39.96\end{array}$ & $\begin{array}{l}57.16 \pm \\
22.64\end{array}$ & $\begin{array}{l}41.95 \pm \\
17.43\end{array}$ & $\begin{array}{l}58.95 \pm \\
19.82\end{array}$ & $\begin{array}{l}85.20 \pm \\
26.28\end{array}$ & $\begin{array}{l}37.72 \pm \\
41.58\end{array}$ & $\begin{array}{l}68.21 \pm \\
17.55\end{array}$ \\
\hline $\begin{array}{l}\text { Disease } \\
\text { duration, y }\end{array}$ & $\leq 1$ & $\begin{array}{l}61.60 \\
\pm \\
29.62 \\
b\end{array}$ & $\begin{array}{l}23.96 \pm \\
39.01^{b}\end{array}$ & $\begin{array}{l}48.36 \pm \\
28.16^{b}\end{array}$ & $\begin{array}{l}39.79 \pm \\
21.43\end{array}$ & $\begin{array}{l}54.17 \pm \\
24.97^{b}\end{array}$ & $\begin{array}{l}80.73 \pm \\
33.76^{b}\end{array}$ & $\begin{array}{l}36.57 \pm \\
44.11\end{array}$ & $\begin{array}{l}62.17 \pm \\
24.60\end{array}$ \\
\hline
\end{tabular}

a Abbreviations for the 8 dimensions of the SF-36: BP, bodily pain; $\mathrm{GH}$, general health; $\mathrm{MH}$, mental health; $\mathrm{PF}$, physical function; RE, role limitations due to emotional problems; RP, role limitations related to physical problems; SF, social functioning; ${ }^{\mathrm{b}} P<0.05 ;{ }^{\mathrm{c}}$ Family income /monthly; ${ }^{d}$ DAS28: clinical remission, <2.6; low, 2.6-3.2; moderate 3.2-5.1; high, > 5.1. 


\begin{tabular}{|c|c|c|c|c|c|c|c|c|c|}
\hline & & PF & RP & BP & $\mathrm{GH}$ & VT & SF & RE & $\mathrm{MH}$ \\
\hline & $1-5$ & $\begin{array}{l}66.06 \\
\pm \\
26.74\end{array}$ & $\begin{array}{l}35.11 \pm \\
42.56\end{array}$ & $\begin{array}{l}55.83 \pm \\
23.92\end{array}$ & $\begin{array}{l}38.40 \pm \\
17.46\end{array}$ & $\begin{array}{l}57.45 \pm \\
20.32\end{array}$ & $\begin{array}{l}84.57 \pm \\
28.10\end{array}$ & $\begin{array}{l}36.17 \pm \\
44.95\end{array}$ & $\begin{array}{l}64.00 \pm \\
20.24\end{array}$ \\
\hline & $>5$ & $\begin{array}{l}50.56 \\
\pm \\
29.45\end{array}$ & $\begin{array}{l}12.15 \pm \\
26.20\end{array}$ & $\begin{array}{l}37.06 \pm \\
23.38\end{array}$ & $\begin{array}{l}32.53 \pm \\
20.76\end{array}$ & $\begin{array}{l}43.40 \pm \\
21.36\end{array}$ & $\begin{array}{l}62.85 \pm \\
29.22\end{array}$ & $\begin{array}{l}22.22 \pm \\
33.10\end{array}$ & $\begin{array}{l}57.50 \pm \\
22.41\end{array}$ \\
\hline \multirow[t]{4}{*}{ DAS28d } & Remission & $\begin{array}{l}82.70 \\
\pm \\
14.82 \\
b\end{array}$ & $\begin{array}{l}58.50 \pm \\
44.50^{b}\end{array}$ & $\begin{array}{l}75.52 \pm \\
13.33^{b}\end{array}$ & $\begin{array}{l}48.92 \pm \\
16.06^{b}\end{array}$ & $\begin{array}{l}65.40 \pm \\
18.59^{b}\end{array}$ & $\begin{array}{l}97.75 \pm \\
24.57^{b}\end{array}$ & $\begin{array}{l}62.00 \pm \\
40.41^{b}\end{array}$ & $\begin{array}{l}71.28 \pm \\
17.50^{b}\end{array}$ \\
\hline & Low & $\begin{array}{l}72.16 \\
\pm \\
18.01\end{array}$ & $\begin{array}{l}20.10 \pm \\
30.01\end{array}$ & $\begin{array}{l}53.47 \pm \\
16.93\end{array}$ & $\begin{array}{l}44.37 \pm \\
19.12\end{array}$ & $\begin{array}{l}61.27 \pm \\
17.60\end{array}$ & $\begin{array}{l}90.93 \pm \\
19.86\end{array}$ & $\begin{array}{l}35.29 \pm \\
43.93\end{array}$ & $\begin{array}{l}69.10 \pm \\
17.78\end{array}$ \\
\hline & Moderate & $\begin{array}{l}49.66 \\
\pm \\
20.75\end{array}$ & $\begin{array}{l}4.31 \pm \\
15.63\end{array}$ & $\begin{array}{l}30.57 \pm \\
16.53\end{array}$ & $\begin{array}{l}29.28 \pm \\
17.84\end{array}$ & $\begin{array}{l}42.16 \pm \\
18.94\end{array}$ & $\begin{array}{l}60.34 \pm \\
25.02\end{array}$ & $\begin{array}{l}10.92 \pm \\
24.49\end{array}$ & $\begin{array}{l}52.48 \pm \\
22.00\end{array}$ \\
\hline & High & $\begin{array}{l}15.16 \\
\pm \\
17.71\end{array}$ & $\begin{array}{l}1.56 \pm \\
6.15\end{array}$ & $\begin{array}{l}15.56 \pm \\
10.81\end{array}$ & $\begin{array}{l}18.91 \pm \\
14.47\end{array}$ & $\begin{array}{l}27.66 \pm \\
19.55\end{array}$ & $\begin{array}{l}40.23 \pm \\
25.55\end{array}$ & $\begin{array}{l}12.50 \pm \\
27.76\end{array}$ & $\begin{array}{l}46.63 \pm \\
26.05\end{array}$ \\
\hline Overall & & $\begin{array}{l}58.53 \\
\pm \\
29.44\end{array}$ & $\begin{array}{l}22.25 \pm \\
36.67\end{array}$ & $\begin{array}{l}45.94 \pm \\
26.37\end{array}$ & $\begin{array}{l}36.71 \pm \\
20.44\end{array}$ & $\begin{array}{l}50.92 \pm \\
23.22\end{array}$ & $\begin{array}{l}74.93 \pm \\
32.05\end{array}$ & $\begin{array}{l}31.06 \pm \\
40.91\end{array}$ & $\begin{array}{l}60.86 \pm \\
22.81\end{array}$ \\
\hline
\end{tabular}

a Abbreviations for the 8 dimensions of the SF-36: BP, bodily pain; $\mathrm{GH}$, general health; $\mathrm{MH}$, mental health; PF, physical function; RE, role limitations due to emotional problems; RP, role limitations related to physical problems; SF, social functioning; ${ }^{\mathrm{b}} P<0.05 ;{ }^{\mathrm{c}}$ Family income /monthly; ${ }^{d}$ DAS28: clinical remission, <2.6; low, 2.6-3.2; moderate 3.2-5.1; high, > 5.1.

For the unstratified (overall) population, the SF-36 scores of the 8 dimensions, from highest to lowest, were social functioning, mental health, physical function, vitality, bodily pain, general health perception, role limitations due to emotional problems, and role limitations related to physical problems. The HRQoL scores of the men and woman were not statistically comparable for each dimension (all $P>0.05$ ). Compared with the younger subgroup (age $<45 \mathrm{y}$ ), the older subgroup had significantly lower scores for physical function, vitality, and social functioning (all $P<0.05$; Table 2).

Among the subgroups based on residence, there were significant differences in the dimensions bodily pain, general health perception, and mental health (all $P<0.05$ ). For subgroups based on monthly family income, significant differences were reported for physical function, bodily pain, vitality, social functioning, and mental health. When stratified by occupation (unemployed, blue, and white collar) and 3 levels of education, each dimension differed significantly among the subgroups except for role limitations due to emotional problems. Significant differences in SF36 scores based on disease duration were found in all dimensions except general health perception, role limitations due to emotional problems, and mental health. When the population was stratified by DAS28 (remission, low, moderate, or high), each dimension differed significantly among these subgroups (Table 2).

\subsection{Association between illness perception and HRQoL}

Based on the linear regression analysis of the SF-36 dimensions, age and disease duration were each significantly associated with every SF-36 dimension, except mental health; and DAS28 was significantly associated with every SF-36 dimension (Table 3). 
Table 3

Linear regression analysis of SF-36 dimensions $(n=191)$

\begin{tabular}{|c|c|c|c|c|c|c|c|c|c|c|}
\hline & & PF & RP & BP & $\mathrm{GH}$ & VT & SF & RE & MH & Total SF-36 \\
\hline \multirow[t]{3}{*}{ Age } & $R^{2}$ & 0.166 & 0.025 & 0.045 & 0.037 & 0.068 & 0.053 & 0.035 & 0.007 & 0.078 \\
\hline & $\beta$ & -0.047 & -0.159 & -0.211 & -0.193 & -0.261 & -0.229 & -0.187 & -0.085 & -0.280 \\
\hline & $P$ & 0.001 & 0.028 & 0.003 & 0.007 & 0.001 & 0.001 & 0.009 & 0.244 & 0.001 \\
\hline \multirow[t]{3}{*}{ Disease duration } & $R^{2}$ & 0.089 & 0.033 & 0.058 & 0.048 & 0.061 & 0.063 & 0.028 & 0.000 & 0.069 \\
\hline & $\beta$ & -0.298 & -0.182 & -0.241 & -0.218 & -0.247 & -0.251 & -0.168 & -0.016 & -0.263 \\
\hline & $P$ & 0.001 & 0.012 & 0.001 & 0.002 & 0.001 & 0.001 & 0.020 & 0.829 & 0.001 \\
\hline \multirow[t]{3}{*}{ DAS28 } & $R^{2}$ & 0.619 & 0.270 & 0.672 & 0.317 & 0.412 & 0.512 & 0.210 & 0.232 & 0.632 \\
\hline & $\beta$ & -0.787 & -0.520 & -0.820 & -0.563 & -0.641 & -0.716 & -0.459 & -0.481 & -0.795 \\
\hline & $P$ & 0.001 & 0.001 & 0.001 & 0.001 & 0.001 & 0.001 & 0.001 & 0.001 & 0.001 \\
\hline \multirow[t]{3}{*}{ BIPQ-1 Consequences } & $R^{2}$ & 0.221 & 0.102 & 0.249 & 0.187 & 0.231 & 0.199 & 0.073 & 0.142 & 0.266 \\
\hline & $\beta$ & -0.470 & -0.319 & -0.499 & -0.433 & -0.481 & -0.446 & -0.270 & -0.377 & -0.516 \\
\hline & $P$ & 0.001 & 0.001 & 0.001 & 0.001 & 0.001 & 0.001 & 0.001 & 0.001 & 0.001 \\
\hline \multirow[t]{3}{*}{ BIPQ-2 Timeline } & $R^{2}$ & 0.055 & 0.083 & 0.111 & 0.100 & 0.057 & 0.085 & 0.066 & 0.013 & 0.114 \\
\hline & $\beta$ & -0.235 & -0.288 & -0.334 & -0.317 & -0.240 & -0.291 & -0.256 & -0.114 & -0.338 \\
\hline & $P$ & 0.001 & 0.001 & 0.001 & 0.001 & 0.001 & 0.001 & 0.001 & 0.115 & 0.001 \\
\hline \multirow[t]{3}{*}{ BIPQ-3 Personal control } & $R^{2}$ & 0.113 & 0.031 & 0.090 & 0.142 & 0.102 & 0.104 & 0.059 & 0.038 & 0.127 \\
\hline & $\beta$ & 0.336 & 0.175 & 0.299 & 0.377 & 0.320 & 0.322 & 0.243 & 0.196 & 0.356 \\
\hline & $P$ & 0.001 & 0.015 & 0.001 & 0.001 & 0.001 & 0.001 & 0.001 & 0.007 & 0.001 \\
\hline \multirow[t]{3}{*}{ BIPQ-4 Treatment control } & $R^{2}$ & 0.006 & 0.014 & 0.002 & 0.023 & 0.010 & 0.007 & 0.025 & 0.004 & 0.018 \\
\hline & $\beta$ & 0.076 & 0.120 & 0.049 & 0.153 & 0.101 & 0.085 & 0.159 & 0.059 & 0.134 \\
\hline & $P$ & 0.298 & 0.097 & 0.499 & 0.035 & 0.165 & 0.242 & 0.028 & 0.414 & 0.066 \\
\hline \multirow[t]{3}{*}{ BIPQ-5 Identity } & $R^{2}$ & 0.257 & 0.174 & 0.300 & 0.173 & 0.221 & 0.244 & 0.138 & 0.126 & 0.330 \\
\hline & $\beta$ & -0.507 & -0.417 & -0.547 & -0.416 & -0.470 & -0.494 & -0.372 & -0.355 & -0.574 \\
\hline & $P$ & 0.001 & 0.001 & 0.001 & 0.001 & 0.001 & 0.001 & 0.001 & 0.001 & 0.001 \\
\hline \multirow[t]{3}{*}{ BIPQ-6 IIIness concern } & $R^{2}$ & 0.008 & 0.020 & 0.023 & 0.028 & 0.021 & 0.007 & 0.017 & 0.006 & 0.025 \\
\hline & $\beta$ & -0.090 & -0.141 & -0.151 & -0.166 & -0.146 & -0.082 & -0.131 & -0.079 & -0.158 \\
\hline & $P$ & 0.216 & 0.052 & 0.037 & 0.022 & 0.044 & 0.261 & 0.071 & 0.276 & 0.029 \\
\hline \multirow[t]{3}{*}{ BIPQ-7 Coherence } & $R^{2}$ & 0.030 & 0.000 & 0.008 & 0.000 & 0.002 & 0.016 & 0.001 & 0.019 & 0.009 \\
\hline & $\beta$ & 0.174 & -0.018 & 0.090 & -0.003 & 0.048 & 0.128 & 0.038 & 0.137 & 0.093 \\
\hline & $P$ & 0.016 & 0.802 & 0.216 & 0.963 & 0.511 & 0.078 & 0.601 & 0.059 & 0.203 \\
\hline \multirow[t]{3}{*}{ BIPQ-8 Emotional response } & $R^{2}$ & 0.235 & 0.158 & 0.276 & 0.245 & 0.270 & 0.282 & 0.165 & 0.266 & 0.376 \\
\hline & $\beta$ & -0.484 & -0.397 & -0.525 & -0.495 & -0.519 & -0.531 & -0.406 & -0.515 & -0.613 \\
\hline & $P$ & 0.001 & 0.001 & 0.001 & 0.001 & 0.001 & 0.001 & 0.001 & 0.001 & 0.001 \\
\hline Overall BIPQ & $R^{2}$ & 0.350 & 0.206 & 0.386 & 0.334 & 0.333 & 0.354 & 0.210 & 0.212 & 0.476 \\
\hline
\end{tabular}




\begin{tabular}{|llllllllll|}
\hline & PF & RP & BP & GH & VT & SF & RE & MH & Total SF-36 \\
\hline$\beta$ & -0.592 & -0.454 & -0.621 & -0.578 & -0.577 & -0.595 & -0.458 & -0.461 & -0.690 \\
\hline$P$ & 0.001 & 0.001 & 0.001 & 0.001 & $\mathbf{0 . 0 0 1}$ & $\mathbf{0 . 0 0 1}$ & $\mathbf{0 . 0 0 1}$ & $\mathbf{0 . 0 0 1}$ & $\mathbf{0 . 0 0 1}$ \\
\hline
\end{tabular}

Note: We use only variables with 0.1 from above and we selection of independent variables for subsequent multiple regression analyses was based on these analyses.

The overall BIPQ score was significantly associated with every SF-36 dimension, as was the following individual BIPQ domains: consequences, personal control, identity, and emotional response. Timeline was significantly associated with every SF-36 dimension, except mental health. Treatment control was associated with general health perception and role limitations due to emotional problems. Illness concern was associated with bodily pain, general health perception, and vitality. Coherence was associated with physical function only. Only the BIPQ domains treatment control and coherence were not significantly associated with the total SF-36 score (Table 3).

To evaluate the effects of certain demographics and illness perceptions on various dimensions of HRQoL in Chinese patients with RA, each HRQoL dimension was taken as a dependent variable, while the general characteristics and all BIPQ dimensions were considered independent variables in the multivariate stepwise regression analysis of HRQoL. According to the results shown in Table 3, only the variables with a significant association $(P<0.1)$ were selected as the independent variables for multivariate stepwise regression (Table 4). Three models were analyzed with different variables as follows: 
Table 4

Multiple linear regression analysis of 4 models $^{a}$ of demographic and illness perceptions by SF-36 HRQoL dimensions ${ }^{b}$

\begin{tabular}{|c|c|c|c|c|c|c|c|c|c|c|}
\hline & & PF & RP & BP & GH & VT & SF & RE & MH & $\begin{array}{l}\text { Total SF- } \\
36\end{array}$ \\
\hline \multirow{8}{*}{$\begin{array}{l}\text { Model } \\
1\end{array}$} & $R^{2}$ & 0.203 & 0.049 & 0.142 & 0.068 & 0.156 & 0.101 & 0.035 & 0.108 & 0.171 \\
\hline & Age & $\begin{array}{l}-0.369 \\
<0.001\end{array}$ & - & $\begin{array}{l}-0.149 \\
0.032\end{array}$ & $\begin{array}{l}-0.158 \\
0.029\end{array}$ & $\begin{array}{l}-0.225 \\
0.001\end{array}$ & $\begin{array}{l}-0.185 \\
0.009\end{array}$ & $\begin{array}{l}-0.187 \\
0.009\end{array}$ & - & $\begin{array}{l}-0.237 \\
0.001\end{array}$ \\
\hline & \multicolumn{10}{|l|}{ Education $^{c}$} \\
\hline & $\begin{array}{l}\text { Senior middle } \\
\text { school }\end{array}$ & - & - & - & - & $\begin{array}{l}0.183 \\
0.007\end{array}$ & - & - & $\begin{array}{l}0.198 \\
0.005\end{array}$ & $\begin{array}{l}0.159 \\
0.019\end{array}$ \\
\hline & College & - & - & - & - & - & - & - & - & - \\
\hline & \multicolumn{10}{|l|}{ Occupation ${ }^{d}$} \\
\hline & Blue collar & - & - & - & - & - & - & - & - & - \\
\hline & White collar & $0.196 ; 0.004$ & $\begin{array}{l}0.222 \\
0.002\end{array}$ & $\begin{array}{l}0.318 \\
<0.001\end{array}$ & $\begin{array}{l}0.178 \\
0.014\end{array}$ & $\begin{array}{l}0.258 \\
<0.001\end{array}$ & $\begin{array}{l}0.225 \\
0.002\end{array}$ & - & $\begin{array}{l}0.285 \\
<0.001\end{array}$ & $\begin{array}{l}0.281 \\
<0.001\end{array}$ \\
\hline \multirow{9}{*}{$\begin{array}{l}\text { Model } \\
2\end{array}$} & $R^{2}$ & 0.646 & 0.270 & 0.700 & 0.317 & 0.437 & 0.524 & 0.210 & 0.284 & 0.650 \\
\hline & Age & $\begin{array}{l}-0.143 \\
0.003\end{array}$ & - & $\begin{array}{l}-0.113 \\
0.010\end{array}$ & - & - & - & - & - & - \\
\hline & \multicolumn{10}{|l|}{ Education $^{c}$} \\
\hline & $\begin{array}{l}\text { Senior middle } \\
\text { school }\end{array}$ & $\begin{array}{l}0.105 \\
0.017\end{array}$ & - & - & - & $\begin{array}{l}0.159 \\
0.004\end{array}$ & $\begin{array}{l}0.111 \\
0.029\end{array}$ & - & $\begin{array}{l}0.198 \\
0.002\end{array}$ & $\begin{array}{l}0.136 \\
0.002\end{array}$ \\
\hline & College & - & - & - & - & - & - & - & - & - \\
\hline & \multicolumn{10}{|l|}{ Occupation ${ }^{d}$} \\
\hline & Blue collar & - & - & - & - & - & - & - & - & - \\
\hline & White collar & - & - & $\begin{array}{l}0.099 \\
0.021\end{array}$ & - & - & - & - & $\begin{array}{l}0.146 \\
0.027\end{array}$ & - \\
\hline & DAS28 & $\begin{array}{l}-0.738 \\
<0.001\end{array}$ & $\begin{array}{l}-0.520 \\
<0.001\end{array}$ & $\begin{array}{l}-0.822 \\
<0.001\end{array}$ & $\begin{array}{l}-0.563 \\
<0.001\end{array}$ & $\begin{array}{l}-0.647 \\
<0.001\end{array}$ & $\begin{array}{l}-0.720 \\
<0.001\end{array}$ & $\begin{array}{l}-0.459 \\
<0.001\end{array}$ & $\begin{array}{l}-0.442 \\
<0.001\end{array}$ & $\begin{array}{l}-0.800 \\
<0.001\end{array}$ \\
\hline \multirow[t]{4}{*}{$\begin{array}{l}\text { Model } \\
3\end{array}$} & $R^{2}$ & 0.670 & 0.305 & 0.722 & 0.425 & 0.499 & 0.573 & 0.266 & 0.314 & 0.728 \\
\hline & Age & $\begin{array}{l}-0.132 \\
0.004\end{array}$ & - & $\begin{array}{l}0.126 \\
0.003\end{array}$ & - & - & - & - & - & - \\
\hline & \multicolumn{10}{|l|}{ Education $^{\mathrm{c}}$} \\
\hline & $\begin{array}{l}\text { Senior middle } \\
\text { school }\end{array}$ & $\begin{array}{l}0.103 \\
0.016\end{array}$ & - & - & $\begin{array}{l}0.112 \\
0.046\end{array}$ & $\begin{array}{l}0.157 \\
0.003\end{array}$ & $\begin{array}{l}0.110 \\
0.023\end{array}$ & - & $\begin{array}{l}0.182 \\
0.003\end{array}$ & $\begin{array}{l}0.135 \\
0.001\end{array}$ \\
\hline
\end{tabular}

a The SF-36 dimensions reflecting HRQoL were treated as dependent variables.

The selection of independent variables for multiple regression analyses was based on these analyses (Table 2 and Table 3 ). Model 1 , demographic variables (gender, age, education, type of residence, family income, employment);

Model 2, Model 1 + clinical characteristic variables (disease duration, DAS28); Model 3, Model 2 + overall BIPQ; Model 4, Model 2 + the 8 BIPQ domains without the overall BIPQ.

beported as: $\beta$; $P$, unless indicated otherwise.

Dummy Variables: ${ }^{c}$ Reference as junior middle school; ${ }^{d}$ Reference as unemployed.

Abbreviations: SF-36 abbreviations: BP, bodily pain; GH, general health; $\mathrm{MH}$, mental health; PF, physical function; RE, role limitations due to emotional problems; RP, role limitations related to physical problems; SF, social functioning. 


\begin{tabular}{|c|c|c|c|c|c|c|c|c|c|c|}
\hline & & PF & RP & BP & GH & VT & SF & RE & MH & $\begin{array}{l}\text { Total SF- } \\
36\end{array}$ \\
\hline & College & - & - & - & - & - & - & - & - & - \\
\hline \multicolumn{11}{|c|}{ Occupation ${ }^{d}$} \\
\hline & Blue collar & - & - & - & - & - & - & - & - & - \\
\hline & White collar & - & - & $\begin{array}{l}0.086 \\
0.038\end{array}$ & - & - & - & - & - & - \\
\hline & DAS28 & $\begin{array}{l}-0.630 \\
<0.001\end{array}$ & $\begin{array}{l}-0.386 \\
<0.001\end{array}$ & $\begin{array}{l}-0.714 \\
<0.001\end{array}$ & $\begin{array}{l}-0.347 \\
<0.001\end{array}$ & $\begin{array}{l}-0.469 \\
<0.001\end{array}$ & $\begin{array}{l}-0.563 \\
<0.001\end{array}$ & $\begin{array}{l}-0.291 \\
<0.001\end{array}$ & $\begin{array}{l}-0.330 \\
<0.001\end{array}$ & $\begin{array}{l}-0.600 \\
<0.001\end{array}$ \\
\hline & Overall BIPQ & $\begin{array}{l}-0.193 \\
<0.001\end{array}$ & $\begin{array}{l}-0.230 \\
0.002\end{array}$ & $\begin{array}{l}-0.215 \\
<0.001\end{array}$ & $\begin{array}{l}-0.379 \\
<0.001\end{array}$ & $\begin{array}{l}-0.307 \\
<0.001\end{array}$ & $\begin{array}{l}-0.270 \\
<0.001\end{array}$ & $\begin{array}{l}-0.290 \\
<0.001\end{array}$ & $\begin{array}{l}-0.272 \\
<0.001\end{array}$ & $\begin{array}{l}-0.343 \\
<0.001\end{array}$ \\
\hline $\begin{array}{l}\text { Model } \\
4\end{array}$ & $R^{2}$ & 0.673 & 0.298 & 0.718 & 0.434 & 0.518 & 0.578 & 0.269 & 0.378 & 0.740 \\
\hline & Age & $\begin{array}{l}-0.145 \\
0.002\end{array}$ & - & $\begin{array}{l}0.129 \\
0.003\end{array}$ & - & - & - & - & - & - \\
\hline \multicolumn{11}{|c|}{ Education ${ }^{c}$} \\
\hline & $\begin{array}{l}\text { Senior middle } \\
\text { school }\end{array}$ & $\begin{array}{l}0.105 \\
0.014\end{array}$ & - & - & $\begin{array}{l}0.113 \\
0.042\end{array}$ & $\begin{array}{l}0.158 \\
0.002\end{array}$ & $\begin{array}{l}0.111 ; \\
0.021\end{array}$ & - & $\begin{array}{l}0.168 \\
0.004\end{array}$ & $\begin{array}{l}0.139 \\
<0.001\end{array}$ \\
\hline & College & - & - & - & - & - & - & - & - & - \\
\hline \multicolumn{11}{|c|}{ Occupation ${ }^{d}$} \\
\hline & Blue collar & - & - & - & - & - & - & - & - & - \\
\hline & White collar & - & - & $\begin{array}{l}0.091 \\
0.030\end{array}$ & - & - & - & - & - & - \\
\hline & DAS28 & $\begin{array}{l}-0.699 \\
<0.001\end{array}$ & $\begin{array}{l}-0.415 \\
<0.001\end{array}$ & $\begin{array}{l}-0.742 \\
<0.001\end{array}$ & $\begin{array}{l}-0.397 \\
<0.001\end{array}$ & $\begin{array}{l}-0.460 \\
<0.001\end{array}$ & $\begin{array}{l}-0.589 \\
<0.001\end{array}$ & $\begin{array}{l}-0.341 \\
<0.001\end{array}$ & $\begin{array}{l}-0.274 \\
<0.001\end{array}$ & $\begin{array}{l}-0.610 \\
<0.001\end{array}$ \\
\hline & $\begin{array}{l}\text { BIPQ-1 } \\
\text { Consequences }\end{array}$ & - & - & - & - & $\begin{array}{l}-0.135 \\
0.032\end{array}$ & - & - & - & - \\
\hline & $\begin{array}{l}\text { BIPQ-3 Personal } \\
\text { control }\end{array}$ & $\begin{array}{l}0.169 \\
<0.001\end{array}$ & - & $\begin{array}{l}0.086 \\
0.038\end{array}$ & $\begin{array}{l}0.225 \\
<0.001\end{array}$ & $\begin{array}{l}0.134 \\
0.014\end{array}$ & $\begin{array}{l}0.138 \\
0.006\end{array}$ & - & - & $\begin{array}{l}0.119 \\
0.004\end{array}$ \\
\hline & $\begin{array}{l}\text { BIPQ-4 Treatment } \\
\text { control }\end{array}$ & - & - & - & - & - & - & $\begin{array}{l}0.139 \\
0.027\end{array}$ & - & $\begin{array}{l}0.084 \\
0.029\end{array}$ \\
\hline & BIPQ-5 Identity & - & $\begin{array}{l}-0.198 \\
0.007\end{array}$ & $\begin{array}{l}-0.146 \\
0.002\end{array}$ & - & - & - & - & - & $\begin{array}{l}-0.105 \\
0.034\end{array}$ \\
\hline & BIPQ-7 Coherence & - & - & - & - & - & - & - & $\begin{array}{l}0.127 \\
0.033\end{array}$ & - \\
\hline & $\begin{array}{l}\text { BIPQ-8 Emotional } \\
\text { response }\end{array}$ & - & - & - & $\begin{array}{l}-0.232 \\
<0.001\end{array}$ & $\begin{array}{l}-0.180 \\
0.006\end{array}$ & $\begin{array}{l}-0.193 \\
0.001\end{array}$ & $\begin{array}{l}-0.224 \\
0.002\end{array}$ & $\begin{array}{l}-0.390 \\
<0.001\end{array}$ & $\begin{array}{l}-0.207 \\
<0.001\end{array}$ \\
\hline \multicolumn{11}{|c|}{ a The SF-36 dimensions reflecting HRQoL were treated as dependent variables. } \\
\hline \multicolumn{11}{|c|}{$\begin{array}{l}\text { The selection of independent variables for multiple regression analyses was based on these analyses (Table } 2 \text { and Table 3). Model 1, } \\
\text { demographic variables (gender, age, education, type of residence, family income, employment); }\end{array}$} \\
\hline \multicolumn{11}{|c|}{$\begin{array}{l}\text { Model 2, Model } 1+\text { clinical characteristic variables (disease duration, DAS28); Model 3, Model } 2+\text { overall BIPQ; Model } 4 \text {, Model } 2+\text { the } 8 \text { BIPQ } \\
\text { domains without the overall BIPQ. }\end{array}$} \\
\hline \multicolumn{11}{|c|}{ beported as: $\beta ; P$, unless indicated otherwise. } \\
\hline \multicolumn{11}{|c|}{ Dummy Variables: ${ }^{c}$ Reference as junior middle school; ${ }^{\mathrm{d}}$ Reference as unemployed. } \\
\hline
\end{tabular}

Model 1 included demographic variables (gender, age, education, type of residence, family income, and employment). Age, education, and occupation could account for $17.1 \%$ of variance of the total SF-36 score (Model $1, \mathrm{R}^{2}=0.171, \mathrm{~F}=12.855, P<0.001$ ). 
Model 2 included the demographic variables of Model 1, plus the clinical characteristics disease duration and DAS28. DAS28 could account for $47.9 \%$ of variance of the total SF-36, in addition to the demographic variables (Model $2, \mathrm{R}^{2}=0.650, \mathrm{~F} \otimes 174.913, P<0.001$ ).

Model 3 included all the variables of Model 2 (demographic variables plus disease duration and DAS28) and the overall BIPQ.

Model 4 incorporated Model 2 and the 8 BIPQ domains, without the overall BIPQ.

In addition, the BIPQ domains personal control, treatment control, identity, and emotional response were associated with HRQoL when the demographic factors and disease characteristics were controlled. The BIPQ domains explained 9.0\% of the variance of the total SF-36 (Model 4 , $\left.\mathrm{R}^{2}=0.740, \mathrm{~F} \otimes 87.37, P<0.001\right)$. Identity and emotional response were negatively associated with HRQL, however personal control and treatment control were positively associated with HRQoL. Furthermore, consequences associated with vitality, and coherence with mental health (Model 4, $P<0.05)$. The total BIPQ had a significant negative association with each component of the SF-36 also the total SF-36 (Model 3 ).

\section{Discussion}

This is the first report of the illness perceptions of patients with RA in China. The overall BIPQ score was $49.09 \pm 11.06$, which was higher than reported by a study from Greece $(40.08 \pm 1.06)$ [18]. This showed that the patients with RA in the present cohort had a more negative view of the disease than did patients in Greece. In western countries, the domain with the highest BIPQ score was timeline, and the lowest was identity[1618]. The present study found that the highest BIPQ score was for illness concern and timeline. Our results showed that the timeline score was higher, in accord with previous studies on RA $[17,18]$. Although the survey populations differed, these study comparisons show that patients with RA in China and in western countries generally recognize that RA is a chronic disease with a long course.

The present study also found that patients older than 45 years had significantly lower scores for physical function, vitality, and social functioning compared with younger patients. Patients with disease duration more than 5 years, and more serious disease activity, had the worst HRQoL. Patients with higher education and engaged in white collar occupations had better HRQoL compared with patients with less education or poorer employment. A literature review reported that increased age was associated with reduced physical function and physical component summary scores for HRQoL[11]. The present analysis also showed that age was negatively associated with physical function, bodily pain, general health perception, vitality, and social functioning.

In addition, multivariate analyses showed that disease activity was negatively associated on each of the SF-36 components. Education and DAS28 were associated with total HRQoL (Model 2, $\mathrm{R}^{2}=0.650, \mathrm{~F} \otimes 174.913, P<0.001$ ). It must be noted that clinical characteristics accounted for $47.9 \%$ of the variance in total HRQoL in addition to the demographic variables (Table 4). These findings revealed that disease activity had a vital effect on the HRQoL of patients with RA. The data in the present study support that increased disease activity is associated with reduced HRQoL in RA[35]. RA has also been noted to affect the HRQoL of patients, by the clinical manifestations of the disease, and by socioeconomic, personal, and environmental factors[36].

There is no doubt that disease activity has an important effect on HRQoL in patients with RA. However, the present results showed that total BIPQ was negatively associated with each of the SF-36 components (Model 3), and domains of BIPQ explained $9.0 \%$ of variance of total SF-36 besides clinical characteristic and demographic variables (Model 4, $\mathrm{R}^{2}=0.740$, F『87.37, $P<0.001$ ).

Hyphantis et al [17] reported that consequences were related to the quality of life of patients with RA. The present study found that consequences were related specifically to vitality in HRQoL. The high scores indicated that patients with strongly held beliefs regarding the serious consequences of RA had worse vitality. Kotsis et al [16] reported that anxiety, depression, and identity were associated with HRQoL in patients with RA, but not personal control or treatment control. However, the present multivariate analysis showed that personal control and treatment control were positively associated with HRQOL, effective treatment and the good self-management of patient ability can control the disease, and improve the HRQoL. The difference in results may be related to differences in the survey population and the survey scales.

A review regarding illness perceptions concluded that negative emotions can affect the treatment of diseases, limit the physiological functions of patients, and seriously affect the prognosis of diseases. Illness perceptions combined with depression, anxiety, and quality of life were closely related[37]. Previous research have shown that depressed patients with RA have poorer long-term outcomes, including increased pain[38], high disease activity[39], increased levels of functional disability, and reduced HRQoL[2].

The present study also found that emotional response in patients with RA was negatively associated with HRQoL (physical function, general health perception, vitality, social functioning, role limitations due to emotional problems, mental health). In addition, identity was negatively associated with HRQoL (role limitations related to physical problems, bodily pain). Our finding emphasized that patients with RA who had serious negative emotions caused by their disease usually had a heavier mental burden and worse quality of life. 
This study specifically showed that different dimensions in illness perceptions influenced different dimensions of HRQoL in patients with RA. The patients with the worst illness perceptions had the worst HRQoL. Previous research in RA showed that illness perceptions had significant implications for adaptation to illness and notably affected medical disease status, even more so than depression, physical function, or pain[40]. Health interventions based on understanding and modifying perceptions of illness was proved useful in facilitating patient's HRQoL[40, 41]. Patients' perspective has great importance and should be valued by clinicians. EULAR recommendations for the management of arthritis suggest that improving the self-management ability of patients with RA will facilitate their health management behavior and improve outcomes[42, 43]. Patients with RA may benefit from illness perception modification. Future evidence-based interventions focusing on illness perception are required to enhance the HRQoL of patients with rheumatoid arthritis[42, 43].

\section{Limitations}

There are some limitations to this study. First, the cases were from a single hospital and the sample size was relatively small. Second, because this is a cross-sectional study, no causal associations could be proved. Longitudinal studies are needed before further conclusions can be drawn. Future studies should add psychological variables such as anxiety and depression, and explore how anxiety, depression, and illness perceptions can affect HRQoL.

\section{Conclusions}

Illness perceptions were associated with the HRQoL of patients with RA in China. Patients with the worst illness perceptions had the worst HRQoL. Illness perceptions are important potential targets to improve the quality of life of patients with RA. This study suggests that enhancing illness perceptions can help improve HRQoL in patients with RA.

\section{Declarations}

\section{Funding}

The National Natural Science Foundation of China (NO. 81773540; NO. 81701617). The National Science and Technology Major Projects of New Drugs (NO.2017ZX10201302-003).

\section{Conflict of interest}

The authors declare that they have no conflict of interest.

\section{Data availability}

The data used are under license for the current study. They are not publicly available.

\section{Ethical approval}

Ethical approval was obtained from the Xijing Hospital Ethics Committee. Ethics approval code: (KY20140902-5).

\section{Informed consent}

Informed consent was obtained from all individual participants included in the study.

\section{Authors' contributions}

All authors contributed to the study conception and design. JW designed the study and wrote the paper, and ZY analyzed the data. JW and ZY contributed equally to this work. ZHZ, LS, and PZ corrected the manuscript, and share corresponding authorship. All authors participated in the questionnaire collection, data input, and read and approved the final version of this manuscript for submission for publication.

\section{References}

1. Smolen JS, Aletaha D, Barton A, Burmester GR, Emery P, Firestein GS, et al. Rheumatoid arthritis. NAT REV DIS PRIMERS. $2018 ; 4: 18001$.

2. Mok CC, Lok EY, Cheung EF. Concurrent psychiatric disorders are associated with significantly poorer quality of life in patients with rheumatoid arthritis. SCAND J RHEUMATOL. 2012;41:253-9.

3. Liu L, Xu X, Xu N, Wang L. Disease activity, resilience and health-related quality of life in Chinese patients with rheumatoid arthritis: a multicenter, cross-sectional study. Health Qual Life Outcomes. 2017;15:149. 
4. Ji J, Zhang L, Zhang Q, Yin R, Fu T, Li L, et al. Functional disability associated with disease and quality-of-life parameters in Chinese patients with rheumatoid arthritis. Health Qual Life Outcomes. 2017;15:89.

5. Zhang N, Fielding R, Soong I, Chan KK, Tsang J, Lee V, et al. Illness perceptions among cancer survivors. SUPPORT CARE CANCER. 2016;24:1295-304.

6. Price A, Goodwin L, Rayner L, Shaw E, Hansford P, Sykes N, et al. Illness perceptions, adjustment to illness, and depression in a palliative care population. J Pain Symptom Manage. 2012;43:819-32.

7. Broadbent E, Petrie KJ, Main J, Weinman J. The brief illness perception questionnaire. J PSYCHOSOM RES. 2006;60:631-7.

8. Hale ED, Treharne GJ, Kitas GD. The common-sense model of self-regulation of health and illness: how can we use it to understand and respond to our patients' needs? Rheumatology. 2007;46:904-6.

9. Petrie KJ, Weinman J. Why illness perceptions matter. Clin Med (Lond). 2006;6:536-9.

10. Chen P, Broadbent E, Coomarasamy C, Jarrett P. Illness perception in association with psychological functioning in patients with discoid lupus erythematosus. Br J Dermatol. 2015;173:824-6.

11. Matcham F, Scott IC, Rayner L, Hotopf M, Kingsley GH, Norton S, et al. The impact of rheumatoid arthritis on quality-of-life assessed using the SF-36: A systematic review and meta-analysis. SEMIN ARTHRITIS RHEU. 2014;44:123-30.

12. Wan SW, He HG, Mak A, Lahiri M, Luo N, Cheung PP, et al. Health-related quality of life and its predictors among patients with rheumatoid arthritis. APPL NURS RES. 2016;30:176-83.

13. Tiemensma J, Gaab E, Voorhaar M, Asijee G, Kaptein AA. Illness perceptions and coping determine quality of life in COPD patients. Int J Chron Obstruct Pulmon Dis. 2016;11:2001-7.

14. Al-Smadi AM, Ashour A, Hweidi I, Gharaibeh B, Fitzsimons D. Illness perception in patients with coronary artery disease: A systematic review. INT J NURS PRACT. 2016;22:633-48.

15. Kaptein AA, Yamaoka K, Snoei L, Kobayashi K, Uchida Y, van der Kloot WA, et al. Illness perceptions and quality of life in Japanese and Dutch patients with non-small-cell lung cancer. LUNG CANCER. 2011;72:384-90.

16. Kotsis K, Voulgari PV, Tsifetaki N, Machado MO, Carvalho AF, Creed F, et al. Anxiety and depressive symptoms and illness perceptions in psoriatic arthritis and associations with physical health-related quality of life. Arthritis Care Res (Hoboken). 2012;64:1593-601.

17. Hyphantis T, Kotsis K, Tsifetaki N, Creed F, Drosos AA, Carvalho AF, et al. The relationship between depressive symptoms, illness perceptions and quality of life in ankylosing spondylitis in comparison to rheumatoid arthritis. CLIN RHEUMATOL. 2013;32:635-44.

18. Kotsis K, Voulgari PV, Tsifetaki N, Drosos AA, Carvalho AF, Hyphantis T. Illness perceptions and psychological distress associated with physical health-related quality of life in primary Sjogren's syndrome compared to systemic lupus erythematosus and rheumatoid arthritis. RHEUMATOL INT. 2014;34:1671-81.

19. Baubet T, Ranque B, Taieb O, Berezne A, Bricou O, Mehallel S, et al. Mood and anxiety disorders in systemic sclerosis patients. PRESSE MED. 2011;40:e111-9.

20. Sharpe L, Sensky T, Allard S. The course of depression in recent onset rheumatoid arthritis: the predictive role of disability, illness perceptions, pain and coping. J PSYCHOSOM RES. 2001;51:713-9.

21. Richards HL, Herrick AL, Griffin K, Gwilliam PD, Loukes J, Fortune DG. Systemic sclerosis: patients' perceptions of their condition. Arthritis Rheum. 2003;49:689-96.

22. Philip EJ, Lindner H, Lederman L. Relationship of illness perceptions with depression among individuals diagnosed with lupus. DEPRESS ANXIETY. 2009;26:575-82.

23. Daleboudt GM, Broadbent E, Berger SP, Kaptein AA. Illness perceptions in patients with systemic lupus erythematosus and proliferative lupus nephritis. LUPUS. 2011;20:290-8.

24. Wilski M, Tasiemski T. Illness perception, treatment beliefs, self-esteem, and self-efficacy as correlates of self-management in multiple sclerosis. ACTA NEUROL SCAND. 2016;133:338-45.

25. Daleboudt GM, Broadbent E, McQueen F, Kaptein AA. The impact of illness perceptions on sexual functioning in patients with systemic lupus erythematosus. J PSYCHOSOM RES. 2013;74:260-4.

26. Nowicka-Sauer K, Hajduk A, Kujawska-Danecka H, Banaszkiewicz D, Smolenska Z, Czuszynska Z, et al. Illness perception is significantly determined by depression and anxiety in systemic lupus erythematosus. LUPUS. 2018;27:454-60.

27. Berner C, Erlacher L, Fenzl KH, Dorner TE. A cross-sectional study on self-reported physical and mental health-related quality of life in rheumatoid arthritis and the role of illness perception. Health Qual Life Outcomes. 2018;16:238.

28. Prevoo ML, van T HM, Kuper HH, van Leeuwen MA, van de Putte LB, van Riel PL. Modified disease activity scores that include twenty-eightjoint counts. Development and validation in a prospective longitudinal study of patients with rheumatoid arthritis. Arthritis Rheum.

1995;38:44-8.

29. Basu S, Poole J. The Brief IIIness Perception Questionnaire. Occup Med (Lond). 2016;66:419-20.

Page $13 / 14$ 
30. Zhang N, Fielding R, Soong I, Chan KK, Lee C, Ng A, et al. Psychometric assessment of the Chinese version of the brief illness perception questionnaire in breast cancer survivors. PLOS ONE. 2017;12:e174093.

31. Wu H, Zhao X, Fritzsche K, Salm F, Leonhart R, Jing W, et al. Negative illness perceptions associated with low mental and physical health status in general hospital outpatients in China. PSYCHOL HEALTH MED. 2014;19:273-85.

32. Ware JJ, Sherbourne CD. The MOS 36-item short-form health survey (SF-36). I. Conceptual framework and item selection. MED CARE. 1992;30:473-83.

33. Li MY, Yang YL, Liu L, Wang L. Effects of social support, hope and resilience on quality of life among Chinese bladder cancer patients: a cross-sectional study. Health Qual Life Outcomes. 2016;14:73.

34. Chen HH, Chen DY, Chen YM, Lai KL. Health-related quality of life and utility: comparison of ankylosing spondylitis, rheumatoid arthritis, and systemic lupus erythematosus patients in Taiwan. CLIN RHEUMATOL. 2017;36:133-42.

35. Kwan YH, Koh ET, Leong KP, Wee HL. Association between helplessness, disability, and disease activity with health-related quality of life among rheumatoid arthritis patients in a multiethnic Asian population. RHEUMATOL INT. 2014;34:1085-93.

36. Ji J, Zhang L, Zhang Q, Yin R, Fu T, Li L, et al. Functional disability associated with disease and quality-of-life parameters in Chinese patients with rheumatoid arthritis. Health Qual Life Outcomes. 2017;15:89.

37. Broadbent E, Wilkes C, Koschwanez H, Weinman J, Norton S, Petrie KJ. A systematic review and meta-analysis of the Brief IIIness Perception Questionnaire. PSYCHOL HEALTH. 2015;30:1361-85.

38. Chancay MG, Guendsechadze SN, Blanco I. Types of pain and their psychosocial impact in women with rheumatoid arthritis. Women's midlife health. 2019;5:3-9.

39. Zhang L, Xia Y, Zhang Q, Fu T, Yin R, Guo G, et al. The correlations of socioeconomic status, disease activity, quality of life, and depression/anxiety in Chinese patients with rheumatoid arthritis. PSYCHOL HEALTH MED. 2017;22:28-36.

40. Groarke A, Curtis R, Coughlan R, Gsel A. The role of perceived and actual disease status in adjustment to rheumatoid arthritis. Rheumatology. 2004;43:1142-9.

41. Dempster M, Howell D, McCorry NK. Illness perceptions and coping in physical health conditions: A meta-analysis. J PSYCHOSOM RES. 2015;79:506-13.

42. Combe B, Landewe R, Lukas C, Bolosiu HD, Breedveld F, Dougados M, et al. EULAR recommendations for the management of early arthritis: report of a task force of the European Standing Committee for International Clinical Studies Including Therapeutics (ESCISIT). ANN RHEUM DIS. 2007;66:34-45.

43. Combe B, Landewe R, Daien $\mathrm{Cl}$, Hua C, Aletaha D, Alvaro-Gracia JM, et al. 2016 update of the EULAR recommendations for the management of early arthritis. ANN RHEUM DIS. 2017;76:948-59. 